\title{
ECG Markers of Hemodynamic Improvement in Patients with Pulmonary Hypertension
}

\author{
Marcin Waligóra (iD, Anna Tyrka, Piotr Podolec, and Grzegorz Kopeć (iD \\ Department of Cardiac and Vascular Diseases, Jagiellonian University Medical College, John Paul II Hospital in Krakow, \\ Pradnicka 80, Kraków, Poland \\ Correspondence should be addressed to Grzegorz Kopeć; grzegorzkrakow1@gmail.com
}

Received 31 October 2017; Revised 2 February 2018; Accepted 27 February 2018; Published 10 April 2018

Academic Editor: Dirk Bandorski

Copyright (C) 2018 Marcin Waligóra et al. This is an open access article distributed under the Creative Commons Attribution License, which permits unrestricted use, distribution, and reproduction in any medium, provided the original work is properly cited.

Introduction. Several diagnostic tests have been recommended for risk assessment in pulmonary hypertension ( $\mathrm{PH})$, but the role of electrocardiography (ECG) in monitoring of PH patients has not been yet established. Therefore the aim of the study was to evaluate which ECG patterns characteristic for pulmonary hypertension can predict hemodynamic improvement in patients treated with targeted therapies. Methods. Consecutive patients with pulmonary arterial hypertension (PAH) or chronic thromboembolic pulmonary hypertension (CTEPH) were eligible to be included if they had had performed two consecutive right heart catheterization (RHC) procedures before and after starting of targeted therapies. Patients were followed up from June 2009 to July 2017. ECG patterns of right ventricular hypertrophy according to American College of Cardiology Foundation were assessed. Results. We enrolled 80 patients with PAH and 11 patients with inoperable CTEPH. The follow-up RHC was performed within $12.6 \pm 10.0$ months after starting therapy. Based on median change of pulmonary vascular resistance, we divided our patients into two subgroups: with and without significant hemodynamic improvement. $R_{V 1}$, $\max R_{V 1,2}+\max S_{\mathrm{I}, \mathrm{aVL}}-S_{V 1}$, and $P_{\mathrm{II}}$ improved along with the improvement of hemodynamic parameters including PVR. They predicted hemodynamic improvement with similarly good accuracy as shown in ROC analysis: $R_{V 1}$ (AUC: 0.75 ; 95\% CI: 0.63-0.84), $P_{\mathrm{II}}$ (AUC: $0.67,95 \%$ CI: $0.56-0.77$ ), and $\max R_{V 1,2}+\max S_{\mathrm{I}, \mathrm{aVL}}-S_{V 1}\left(0.73 ; 95 \% \mathrm{CI}\right.$ : 0.63-0.82). In Cox regression only change in $R_{V 1}$ remained significant mortality predictor (HR: 1.12, 95\% CI: 1.01-1.24). Conclusion. Electrocardiogram may be useful in predicting hemodynamic effects of targeted therapy in precapillary pulmonary hypertension. Decrease of $R_{V 1}$, $\max R_{V 1,2}+\max S_{\mathrm{I}, \mathrm{aVL}}-S_{V 1}$, and $P_{\mathrm{II}}$ corresponds with hemodynamic improvement after treatment. Of these changes a decrease of $R$ wave amplitude in $V_{1}$ is associated with better survival.

\section{Introduction}

Pulmonary hypertension $(\mathrm{PH})$ is a severe progressive disease characterized by narrowing and occlusion of pulmonary arteries. The current treatment algorithm requires frequent assessment of the patient and escalation of therapy if low risk status has not been achieved. Several diagnostic tests have been recommended for risk evaluation including physical examination, assessment of World Heart Organization functional class (WHO-FC), 6-minute walk test (6 MWT), cardiopulmonary exercise testing, N-terminal pro-B type natriuretic peptide level (NT-proBNP), imaging studies, and right heart catheterization (RHC) [1]. Till now the role of electrocardiography (ECG) in monitoring of $\mathrm{PH}$ patients has not been established.
Chronic overload of the right ventricle (RV) as observed in $\mathrm{PH}$ leads to RV hypertrophy and dilation. This can be reflected by several patterns in surface electrocardiogram. Hemodynamic, autopsy, and cardiac magnetic resonance imaging (cMR) studies have shown significant correlations between hemodynamic burden, right ventricular hypertrophy, and ECG changes as reported in several studies [29]. Little is known however about whether hemodynamic improvement observed in treated $\mathrm{PH}$ patients can be reflected by ECG changes.

Therefore the aim of the study was to evaluate which ECG patterns characteristic for pulmonary hypertension can predict hemodynamic improvement in patients treated with targeted therapies. 


\section{Methods}

2.1. Patients. All study participants were selected from a cohort of patients with $\mathrm{PH}$ diagnosed and treated in our centre between June 2009 and March 2016. Patients were eligible if they had pulmonary arterial hypertension (PAH) or inoperable chronic thromboembolic pulmonary hypertension (CTEPH) with RHC and ECG performed on the same day at least twice: the first before starting or escalating targeted therapy and the second at least 3 months later. Pulmonary hypertension was defined as a mean pulmonary artery pressure (mPAP) $\geq 25 \mathrm{mmHg}$ at rest as assessed by right heart catheterization. PAH was defined as precapillary pulmonary hypertension (pulmonary artery wedge pressure $\leq 15 \mathrm{mmHg}$ ) with pulmonary vascular resistance $>3$ Wood units in the absence of other causes of precapillary $\mathrm{PH}$ such as lung diseases, chronic thromboembolic pulmonary hypertension, or other rare diseases [1]. CTEPH was defined as precapillary pulmonary hypertension (pulmonary artery wedge pressure $\leq 15 \mathrm{mmHg}$ ) after at least 3 months of effective anticoagulation with a presence of mismatched perfusion defects on lung scan or multidetector CT angiography [1]. The operability assessment in all CTEPH patients was performed by a multidisciplinary CTEPH team. Both treatment-naive patients (patients who have not been previously treated with therapies specific for pulmonary arterial hypertension) and patients already treated with PAH targeted medications could have been included. Main exclusion criteria were age $<18$ years and lack of informed consent. Clinical assessment included demographic information, patient's medical history, NT-proBNP, $6 \mathrm{MWT}$ distance (6 MWD), assessment of the WHO-FC, resting 12-lead ECG, and RHC. Patients were treated with $\mathrm{PAH}$ specific drugs according to European Society of Cardiology (ESC) guidelines [1] and local standards. All-cause mortality was ascertained by data collection (1) from medical registry of hospital, (2) from the Department of Nationals' and Foreigners' Affairs, or (3) through phone follow-up. Patients were enrolled between June 2009 and March 2016 and the observation period was extended until July 2017. The baseline assessment was at the time of RHC which resulted in diagnosis of $\mathrm{PH}$ or escalation of targeted therapy. The study protocol conforms to the ethical guidelines of the 1975 Declaration of Helsinki and was approved by the institutional ethics committee. Informed consent was obtained from each patient before starting the study.

2.2. Electrocardiography. A 12-lead standard ECG $(10 \mathrm{~mm}=$ $1 \mathrm{mV}, 25 \mathrm{~mm} / \mathrm{s}$ ) was acquired in a supine position during quiet respiration. For the purpose of the present study we assessed several parameters proposed by American College of Cardiology Foundation and the Heart Rhythm Society (AHA/ACCF/HRS) [10] to diagnose RV hypertrophy. We assessed quantitative parameters: $R_{V 1}, R: S_{V 1}, S_{V 5}, S_{V 6}, R_{\mathrm{aVR}}$, $S_{V 1}, R_{V 5,6}, R: S_{V 5}, R: S_{V 6}, R: S_{V 5}$ to $R: S_{V 1},\left(R_{\mathrm{I}}+S_{\mathrm{III}}\right)-\left(S_{\mathrm{I}}+\right.$ $R_{\mathrm{III}}$ ), $\max R_{V 1,2}+\max S_{\mathrm{I}, \mathrm{aVL}}-S_{V 1}, R_{V 1}+S_{V 5,6}, R$ peak $V_{1}$ (QRS duration $<0.12 \mathrm{sec}$ ), and $P_{\mathrm{II}}$, as well as qualitative patterns: presence of QR in $V_{1}, \mathrm{RSR}_{V 1}$ (QRS duration $>0.12 \mathrm{sec}$ ), $S>$ $R_{\text {I,II,III }}, S_{\text {I }}$ and $Q_{\text {III }}, R: S_{V 1}>R: S_{V 3,4}$, and negative $T$-waves in leads $V_{1}-V_{3}$. Right bundle branch block (RBBB) was as recommended [11]: QRS duration $\geq 120 \mathrm{~ms}$, with typical QRS morphology in $V_{1}$ or $V_{2}$ (rsr', rsR', rSR', wide and notched $R$ ), and $S$ wave duration $>R$ wave duration or $>40 \mathrm{~ms}$ in $\mathrm{I}$ and $V_{6}$. When a pure dominant $R$ wave with or without a notch was present in $V_{1}$, addition criterion had to be satisfied: normal $R$ peak time in $V_{5}$ and $V_{6}$ but $>50 \mathrm{~ms}$ in $V_{1}$.

2.3. Right Heart Catheterization. RHC was performed in a supine position from the right femoral vein or right internal jugular vein access using a Swan-Ganz catheter. All measurements including acquisition of pressure waves were made at end expiration. Cardiac output was measured using the Fick direct oxygen consumption method. Blood oxygen saturation was measured with CO-oximeter OSM3 (Radiometer, Copenhagen, Denmark). Cardiac index (CI) was calculated as cardiac output divided by body surface area (BSA). BSA was calculated from the Mosteller formula [12]. Pulmonary vascular resistance (PVR) was calculated as the difference between mean pulmonary arterial pressure (mPAP) and pulmonary artery wedge pressure divided by cardiac output. Based on a median change of PVR in all study patients we distinguished two subgroups: with and without hemodynamic improvement.

2.4. Statistics. Continuous variables are reported using means and standard deviations. Categorical variables are described as counts and percentages. Continuous variables were compared using the Student $t$-test or Mann-Whitney $U$ test when appropriate. The $\chi^{2}$-test was used to compare categorical variables. McNemar's test was used to compare paired data of meeting right ventricular hypertrophy criteria before and after addition of targeted therapy. The Bonferroni correction was applied when changes in several ECG patterns were compared between patients with and without hemodynamic improvement. The relationship between changes of ECG and hemodynamics was estimated by Pearson or Spearman correlation tests. Several receiver-operating characteristics (ROC) curves were drawn to compare the accuracy of changes in different ECG patterns in predicting hemodynamic improvement. Univariate Cox regression analysis was used to assess significant associations between changes in ECG and survival. Statistical analysis was performed with Statistica PL software [Dell Inc. (2016), Dell Statistica (data analysis software system), version 13; software.dell.com] and MedCalc Statistical software version 16.8 (MedCalc software bvba, Ostend, Belgium; https://www.medcalc.org; 2016). The significance level was set at alpha level of 0.05 .

\section{Results}

3.1. Patients. Between June 2009 and March 2016, 158 patients were diagnosed with PAH and 46 with CTEPH. Among them 80 patients with $\mathrm{PAH}$ and 11 with inoperable CTEPH were included in the present analysis. Excluded PAH patients did not have follow-up RHC (Eisenmenger's syndrome; $n=55$, lack of consent; $n=2$, lost to follow-up; $n=1$, premature death; $n=12)$, permanent pacemaker stimulation $(n=$ $5)$, or insufficient quality of ECG recordings $(n=3)$. We 
excluded the CTEPH patients who were referred to pulmonary endarterectomy or pulmonary balloon angioplasty.

Overall the study sample included 91 patients aged $52.6 \pm$ 16.4 (68.1\% females). The patients had idiopathic PAH (IPAH; $n=54,59.3 \%)$, PAH associated with connective tissue disease CTD-APAH ( $n=16,17.6 \%)$, and PAH associated with congenital heart diseases (CHD-APAH; $n=10,11 \%$ ) or inoperable CTEPH $(n=11,12.1 \%)$. Patients were in WHOFC II $(n=9,9.9 \%)$, III $(n=61 ; 67 \%)$, or IV $(n=21,23.1 \%)$ at initial assessment. Majority of patients were newly diagnosed and treatment-naive $(n=71,78 \%)$ and were initially treated with a monotherapy of either phosphodiesterase- 5 inhibitor (PDE-5i) in 35 (49.2\%), endothelin receptor antagonist (ERA) in $14(17.7 \%)$, parenteral prostacyclin analogue in $10(14.1 \%)$, inhaled iloprost 4 (5.6\%), or calcium channel blocker in 8 $(11.3 \%)$. The other patients $(n=20)$ were already treated with targeted therapies: riociguat, 1 (1.1\%); ERA, 4 (4.4\%); PDE-5i, 9 (9.9\%); PDE-5i and ERA, 4 (4.4\%); PDE-5i and inhaled iloprost, 1 (1.1\%); and inhaled iloprost and ERA, 1 (1.1\%). In this group follow-ups were gathered after addition of the following treatment: parenteral prostacyclin analogue in $17(85 \%)$, PDE-5i in $1(5 \%)$, ERA in $1(5 \%)$, and inhaled iloprost in $1(5 \%)$.

The follow-up RHC was performed within $12.6 \pm 10.0$ months after study enrollment. The median change of pulmonary vascular resistance was $-2.1[-4.5 ; 0.7]$ Wood Units which was $17 \%$ of the baseline PVR value. Based on this value, we divided our patients into two groups: with significant hemodynamic improvement (decrease of PVR $\geq 17 \%$ from baseline value, $n=46$ ) and without significant hemodynamic improvement (decrease of PVR $<17 \%$ from baseline value, $n=46$ ). Baseline clinical and hemodynamic characteristics of these two subgroups were similar as shown in Table 1. Patients with hemodynamic improvement had added parenteral prostacyclin analogues more frequently than patients without hemodynamic improvement [18 (39.1\%) versus 9 $(20 \%), p=0.05]$. No differences were observed with reference to other therapies: PDE-5 inhibitors [16 (34.5\%) versus 20 (44.4\%), $p=0.35$ ], ERA [5 (10.9\%) versus 10 (22.2\%), $p=0.15$ ], inhaled iloprost [3 (6.5\%) versus 2 (4.4\%) versus, $p=0.67$ ], or CCB [4 (8.7\%) versus $(8.9 \%)$, $p=0.97]$. After addition of specific treatment we found the following changes of hemodynamic parameters in a group with and without hemodynamic improvement, respectively: $\mathrm{mPAP}-8.7 \pm 10.8 \mathrm{mmHg}(p<0.001)$ and $+0.8 \pm 11.3 \mathrm{mmHg}$ $(p=0.64)$, cardiac index $+0.59 \pm 0.91 / \mathrm{kg} / \mathrm{m} 2(p<0,001)$ and $-0.14 \pm 0.561 / \mathrm{kg} / \mathrm{m} 2(p=0.1)$, mRAP $-2.5 \pm 5.5 \mathrm{mmHg}$ $(p=0.02)$ and $-0.9 \pm 4,2 \mathrm{mmHg}(p=0.45)$, and PVR $-5.4 \pm 4.5 \mathrm{WU}(p<0.01)$ and $+1.9 \pm 4.8 \mathrm{WU}(p=0.01)$.

3.2. ECG Changes after Treatment. Patients without complete or incomplete RBBB accounted for majority of the study group $(n=74,81.3 \%)$. Twelve patients presented atrial fibrillation during initial assessment; therefore they did not have $P$ wave amplitudes calculated (6 in group with significant hemodynamic improvement and 6 in group without it). Twenty-four patients had no $S_{V 1}$ and were not eligible for calculations of criteria using $S$ wave. Baseline electrocardiographic characteristics of patients with and without hemodynamic improvement are showed in Table 2. The follow-up ECG showed that after addition of PAH specific therapy in the whole sample none of ECG parameters changed significantly. However when sample was divided into patients with and without hemodynamic improvement we found differences in changes of the following parameters: $R_{V 1}, \max R_{V 1,2}+\max S_{\mathrm{I}, \mathrm{aVL}}-S_{V 1}$, and $P_{\mathrm{II}}$ as shown in Table 3 and Table S1. Changes of fulfilling these RVH criteria before and after addition of targeted treatment are presented in Figure 1. Similar observations were also present when only patients without RBBB were assessed as presented in Tables S2 and S3. Changes in these ECG patterns correlated with changes of several hemodynamic parameters including $\triangle \mathrm{PVR}, \triangle \mathrm{mPAP}$, and $\triangle \mathrm{CI}$ as shown in Table 4 . In Figure 2. we compare three ROC analyses to show how changes in different ECG parameters predicted significant hemodynamic improvement in ROC analysis $(p=0.56$ for comparison of $\Delta R_{V 1}$ and $\Delta \max R_{V 1,2}+\max S_{\mathrm{I}, \mathrm{aVL}}-S_{V 1}, p=0.4$ for comparison of $\Delta R_{V 1}$ and $\Delta P_{\mathrm{II}}$, and $p=0.18$ for comparison of $\Delta \max R_{V 1,2}+\max S_{\mathrm{I}, \mathrm{aVL}}-S_{V 1}$ and $\left.\Delta P_{\mathrm{II}}\right)$.

The RBBB was diagnosed in 17 patients at baseline assessment (complete in 9 and incomplete in 8 patients). In this group we did not find any differences in changes of ECG patterns with and without hemodynamic improvement as presented in Tables S4 and S5.

3.3. Electrocardiography Changes and Long-Term Follow-Up. During prospective observation of a mean of $27.9 \pm 9.5$ months, 20 patients died (22.0\%). In univariate Cox proportional hazard models, change in $R_{V 1}$ was significantly associated with mortality (HR: 1.12, 95\% CI: 1.01-1.24, $p=$ 0.02). Changes in the other criteria were not significantly associated with mortality. At follow-up $R_{V 1}$ increased in $36(39.6 \%)$ patients, decreased in $48(52.7 \%)$ patients, and remained unchanged in 7 (7.7\%) patients. We observed 12 (33.3\%), $7(14.6 \%)$, and $1(14.3 \%)$ deaths in the respective groups.

\section{Discussion}

In the present study we showed that hemodynamic improvement in PAH patients treated with targeted therapy is reflected by favorable changes in several ECG patterns. This referred only to the group without RBBB. Additionally we showed that in this group of patients a change in amplitude of $R$ wave in $V_{1}$ after targeted treatment is associated with longterm survival.

4.1. Electrocardiographic Signs of Pulmonary Hypertension. Structural changes in right atrium and right ventricle such as hypertrophy or dilation as observed in $\mathrm{PH}$ are reflected by several ECG patterns [2,13-20]. Some of them including qR in lead $V_{1}$ [21-23], $p$ wave amplitude in lead II [21], resting heart rate [24], $p$ wave duration [25], precordial electrocardiogram voltage (sum of $R$ wave in $V_{1}$ and maximum $S$ wave amplitude in $V_{5}$ or $V_{6}$ ) [26], QRS duration [27], and QTc duration [28] have been shown to have prognostic impact in patients with IPAH, ES, or CTEPH. Despite that, 


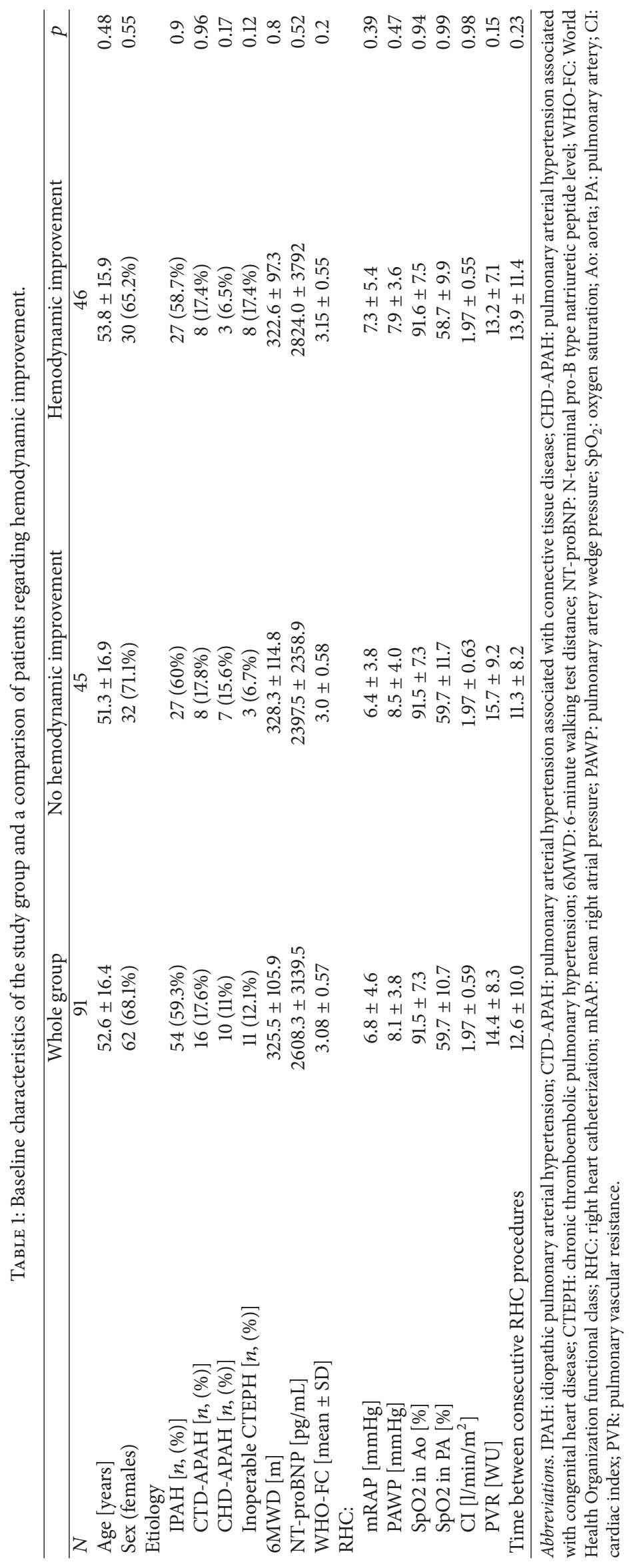


TABLE 2: Baseline electrocardiography characteristics.

\begin{tabular}{|c|c|c|c|}
\hline & $\begin{array}{c}\text { No hemodynamic } \\
\text { improvement }\end{array}$ & $\begin{array}{l}\text { Hemodynamic } \\
\text { improvement }\end{array}$ & $p$ \\
\hline HR [bpm] & $79.0 \pm 17.2$ & $76.4 \pm 16.4$ & 0.46 \\
\hline Sinus rhythm $[n(\%)]$ & $39(86.7 \%)$ & $40(87.0 \%)$ & 0.97 \\
\hline$R_{V 1}[\mathrm{~mm}]$ & $6.5 \pm 5.7$ & $4.2 \pm 3.0$ & 0.02 \\
\hline$R_{V 1}>6 \mathrm{~mm}[n(\%)]$ & $17(37.8 \%)$ & $13(28.3 \%)$ & 0.34 \\
\hline$R: S_{V 1}[\mathrm{~mm}]^{1}$ & $6.9 \pm 12.0$ & $3.2 \pm 4.6$ & 0.09 \\
\hline$R: S_{V 1}>1.0[n(\%)]$ & $36(80 \%)$ & $29(63 \%)$ & 0.08 \\
\hline$S_{V 5}[\mathrm{~mm}]$ & $9.5 \pm 5.5$ & $8.6 \pm 4.5$ & 0.39 \\
\hline$S_{V 5}>10 \mathrm{~mm}[n(\%)]$ & $17(37.8 \%)$ & $17(37 \%)$ & 0.94 \\
\hline$S_{V 6}[\mathrm{~mm}]$ & $6.5 \pm 4.6$ & $6.1 \pm 3.8$ & 0.64 \\
\hline$S_{V 6}>3 \mathrm{~mm}[n(\%)]$ & $33(73.3 \%)$ & $35(76 \%)$ & 0.76 \\
\hline$R_{\mathrm{aVR}}[\mathrm{mm}]$ & $3.2 \pm 2.3$ & $2.5 \pm 2.1$ & 0.17 \\
\hline$R_{\mathrm{aVR}}>4 \mathrm{~mm}[n(\%)]$ & $9(20 \%)$ & $5(10.9 \%)$ & 0.62 \\
\hline$S_{V 1}[\mathrm{~mm}]$ & $2.6 \pm 4.0$ & $2.8 \pm 3.4$ & 0.78 \\
\hline$S_{V 1}<2 \mathrm{~mm}[n(\%)]$ & $29(64.4 \%)$ & $26(56.5 \%)$ & 0.44 \\
\hline$R_{V 5,6}[\mathrm{~mm}]$ & $7.9 \pm 4.3$ & $7.7 \pm 3.3$ & 0.83 \\
\hline$R_{V 5,6}<3 \mathrm{~mm}[n(\%)]$ & $3(6.7 \%)$ & $3(6.5 \%)$ & 0.98 \\
\hline$R: S_{V 5}[\mathrm{~mm}]$ & $1.3 \pm 1.0$ & $1.8 \pm 2.7$ & 0.25 \\
\hline$R: S_{V 5}<3 \mathrm{~mm}[n(\%)]$ & $15(33.3 \%)$ & $12(26 \%)$ & 0.45 \\
\hline$R: S_{V 6}[\mathrm{~mm}]$ & $1.9 \pm 1.9$ & $2.7 \pm 4.5$ & 0.31 \\
\hline$R: S_{V 6}<3 \mathrm{~mm}[n(\%)]$ & $1(2.2 \%)$ & $4(8.7 \%)$ & 0.18 \\
\hline$R: S_{V 5}$ to $R: S_{V 1}[\mathrm{~mm}]^{1}$ & $3.0 \pm 6.4$ & $6.4 \pm 19.4$ & 0.35 \\
\hline$R: S_{V 5}$ to $R: S_{V 1}<0.04[n(\%)]^{1}$ & $1(2.2 \%)$ & $1(2.2 \%)$ & 1 \\
\hline$\left(R_{\mathrm{I}}+S_{\mathrm{III}}\right)-\left(S_{\mathrm{I}}+R_{\mathrm{III}}\right)[\mathrm{mm}]$ & $-8.8 \pm 9.5$ & $-5.3 \pm 9.9$ & 0.1 \\
\hline $\begin{array}{l}\left(R_{\mathrm{I}}+S_{\mathrm{III}}\right)-\left(S_{\mathrm{I}}+R_{\mathrm{III}}\right)<15 \mathrm{~mm} \\
{[n(\%)]}\end{array}$ & $44(97.8 \%)$ & $45(97.8 \%)$ & 0.99 \\
\hline $\begin{array}{l}\max R_{V 1,2}+\max S_{\mathrm{I}, \mathrm{aVL}}-S_{V 1} \\
{[\mathrm{~mm}]}\end{array}$ & $13.5 \pm 11.3$ & $9.2 \pm 7.2$ & 0.03 \\
\hline $\begin{array}{l}\max R_{V 1,2}+\max S_{\mathrm{I}, \mathrm{aVL}}-S_{V 1}> \\
6 \mathrm{~mm}[n(\%)]\end{array}$ & $36(80 \%)$ & $37(80.4 \%)$ & 0.96 \\
\hline$R_{V 1}+S_{V 5,6}[\mathrm{~mm}]$ & $16.3 \pm 9.7$ & $12.8 \pm 6.1$ & 0.05 \\
\hline$R_{V 1}+S_{V 5,6}>10.5[n(\%)]$ & $33(73.3 \%)$ & $29(63 \%)$ & 0.29 \\
\hline$R_{V 1}$ peak $[\mathrm{mm}]$ & $40.5 \pm 13.8$ & $38.8 \pm 20.6$ & 0.54 \\
\hline $\begin{array}{l}R \text { peak } V_{1}>35 \text { msec in baseline }{ }^{1} \\
{[n(\%)]}\end{array}$ & $21(65.6 \%)$ & $21(61.2 \%)$ & 0.75 \\
\hline$P_{\mathrm{II}}[\mathrm{mm}]^{3}$ & $1.48 \pm 0.6$ & $1.7 \pm 0.8$ & 0.29 \\
\hline$P_{\mathrm{II}}>0,25 \mathrm{mV}[n(\%)]^{3}$ & $1(2.6 \%)$ & $5(12.2 \%)$ & 0.11 \\
\hline \multicolumn{4}{|l|}{ Qualitative patterns: } \\
\hline $\mathrm{qR}$ in $V_{1}[n(\%)]$ & $21(46.7 \%)$ & $17(40 \%)$ & 0.35 \\
\hline $\mathrm{RSR}_{V 1}(\mathrm{QRS}$ duration $>0.12 \mathrm{sec})$ & $3(6.7 \%)$ & $4(8.7 \%)$ & 0.72 \\
\hline$S>R$ in $\mathrm{I}$ & $34(75.6 \%)$ & $26(56.5 \%)$ & 0.06 \\
\hline$S>R$ in II & $12(26.7 \%)$ & $8(17.4 \%)$ & 0.29 \\
\hline$S>R$ in III & $7(15.6 \%)$ & $9(15.6 \%)$ & 0.62 \\
\hline$S_{\mathrm{I}}$ and $Q_{\mathrm{III}}$ & $25(55.6 \%)$ & $26(56.5 \%)$ & 0.93 \\
\hline$R: S_{V 1}>R: S_{V 3,4}$ & $20(64.5 \%)$ & $20(55.6 \%)$ & 0.46 \\
\hline Negative $T$-wave $V_{1}$ through $V_{3}$ & $25(55.6 \%)$ & $27(58.7 \%)$ & 0.76 \\
\hline
\end{tabular}

${ }^{1}$ Calculated for patients who had all required waves present; ${ }^{2}$ calculated according to guidelines, only for patients with QRS $<120 \mathrm{msec} ; n=32$ and $n=34$, respectively; ${ }^{3}$ calculated only for patients in sinus rhythm; $n=39$ and $n=40$, respectively. 
TABLE 3: Changes of quantitative electrocardiographic parameters after PAH specific treatment.

\begin{tabular}{|c|c|c|c|}
\hline & $\begin{array}{c}\text { No hemodynamic } \\
\text { improvement }\end{array}$ & $\begin{array}{c}\text { Hemodynamic } \\
\text { improvement }\end{array}$ & $p^{*}$ \\
\hline$\Delta R_{V 1}[\mathrm{~mm}]$ & $+1.38 \pm 3.9$ & $-0.82 \pm 2.0$ & 0.002 \\
\hline$\Delta R: S_{V 1}[\mathrm{~mm}]$ & $-1.0 \pm 4.9^{1}$ & $-0.6 \pm 1.4^{1}$ & 1 \\
\hline$\Delta S_{V 5}[\mathrm{~mm}]$ & $+0.17 \pm 4.7$ & $-1.1 \pm 4.0$ & 1 \\
\hline$\Delta S_{V 6}[\mathrm{~mm}]$ & $+1.3 \pm 5.6$ & $-0.91 \pm 3.9$ & 0.45 \\
\hline$\Delta R_{\mathrm{aVR}}[\mathrm{mm}]$ & $-0.1 \pm 2.0$ & $-0.7 \pm 1.9$ & 1 \\
\hline$\Delta S_{V 1}[\mathrm{~mm}]$ & $-0.46 \pm 3.0$ & $-0.48 \pm 2.7$ & 1 \\
\hline$\Delta R_{V 5,6}[\mathrm{~mm}]$ & $-0.7 \pm 4.4$ & $0 \pm 3.1$ & 1 \\
\hline$\Delta R: S_{V 5}[\mathrm{~mm}]$ & $-0.2 \pm 1.2$ & $+0.2 \pm 1.2$ & 1 \\
\hline$\Delta R: S_{V 6}[\mathrm{~mm}]$ & $-0.8 \pm 1.9$ & $+0.3 \pm 2.8$ & 0.6 \\
\hline$\Delta R: S_{V 5}$ to $R: S_{V 1}[\mathrm{~mm}]$ & $-1.6 \pm 4.0^{1}$ & $+1.2 \pm 4.9^{1}$ & 1 \\
\hline$\Delta\left(R_{\mathrm{I}}+S_{\mathrm{III}}\right)-\left(S_{\mathrm{I}}+R_{\mathrm{III}}\right)[\mathrm{mm}]$ & $-2.1 \pm 6.9$ & $+0.9 \pm 4.1$ & 0.2 \\
\hline$\Delta \max R_{V 1,2}+\max S_{\mathrm{I}, \mathrm{aVL}}-S_{V 1}[\mathrm{~mm}]$ & $+3.4 \pm 7.4$ & $-1.0 \pm 4.6$ & 0.002 \\
\hline$\Delta R_{V 1}+S_{V 5,6}[\mathrm{~mm}]$ & $+1.7 \pm 6.7$ & $-1.9 \pm 4.3$ & 0.06 \\
\hline$\Delta R$ peak $V_{1}[\mathrm{~mm}]$ & $-2.9 \pm 22.2^{2}$ & $-11.3 \pm 23.4^{2}$ & 1 \\
\hline$\Delta P_{\mathrm{II}}$ & $+0.5 \pm 1.0$ & $-0.1 \pm 0.7$ & 0.03 \\
\hline
\end{tabular}

${ }^{1}$ Calculated for patients who had all required waves present; ${ }^{2}$ calculated according to guidelines, only for patients with QRS $<120 \mathrm{msec} ; n=32$ and $n=34$, respectively. ${ }^{*} p$ values were mathematically adjusted using Bonferroni correction for multiple comparisons.

$\begin{array}{lllllllllll}0.0 & 10.0 & 20.0 & 30.0 & 40.0 & 50.0 & 60.0 & 70.0 & 80.0 & 90.0 & 100.0\end{array}$

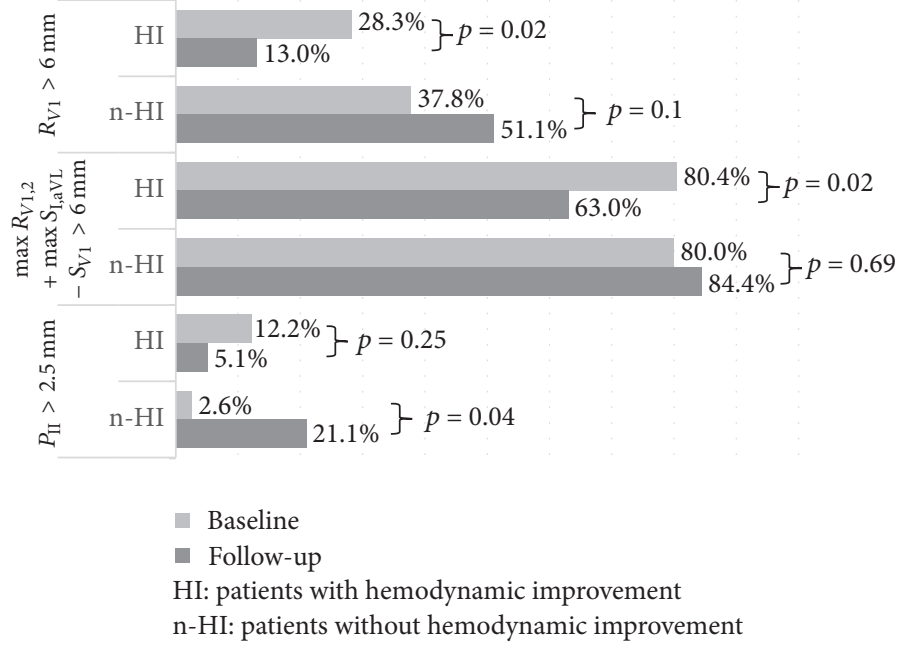

FIGURE 1: Changes of fulfilling right ventricular hypertrophy criteria in a study group before and after addition of targeted treatment.

little is known about dynamics of ECG changes during the course of the disease. In a single study of Tonelli et al. [29] electrocardiograms were assessed at time of diagnosis of pulmonary hypertension and before death. The study showed that some ECG parameters altered as the disease progressed. There was an increase in median heart rate; $R$ : $S_{V 1}$ ratio; and duration of PR interval, QRS complex, and QTc. Favorable dynamics of electrocardiogram was also shown in our study, but only in the group of patients with significant hemodynamic improvement. On the other hand, patients in whom we did not observe hemodynamic improvement ECG parameters worsened.
4.2. Correlations between ECG and Hemodynamics in PAH. Only few studies have evaluated the relationship between surface ECG and hemodynamics in PH. The first study was conducted before the era of PAH specific treatment by Kanemoto in 47 patients with IPAH [8]. In that study the amplitude of $R_{V 1}$ and $R: S_{V 1}$ correlated with the pulmonary artery systolic pressure $(r=0.46, p<0.01$, and $r=$ $0.50, p<0.01$, resp.), and an amplitude of $R_{V 1}>1.2 \mathrm{mV}$ indicated a pulmonary artery systolic pressure of more than $90 \mathrm{mmHg}$ with a sensitivity of $94 \%$ and a specificity of $47 \%$. Additionally, amplitude of the $R_{V 5}(r=0.46, p<0.01), R_{V 6}$ $(r=0.46, p<0.01), R: S_{V 5}(r=0.39, p<0.01)$, and $R: S_{V 6}$ 
TABLE 4: Correlation between changes in ECG criteria and hemodynamic variables.

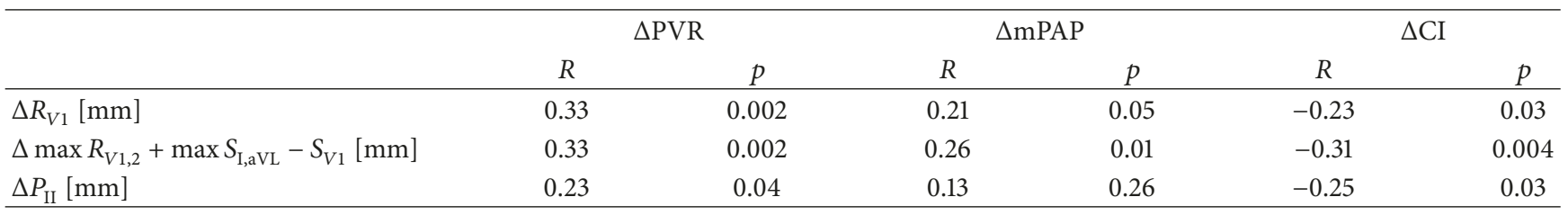

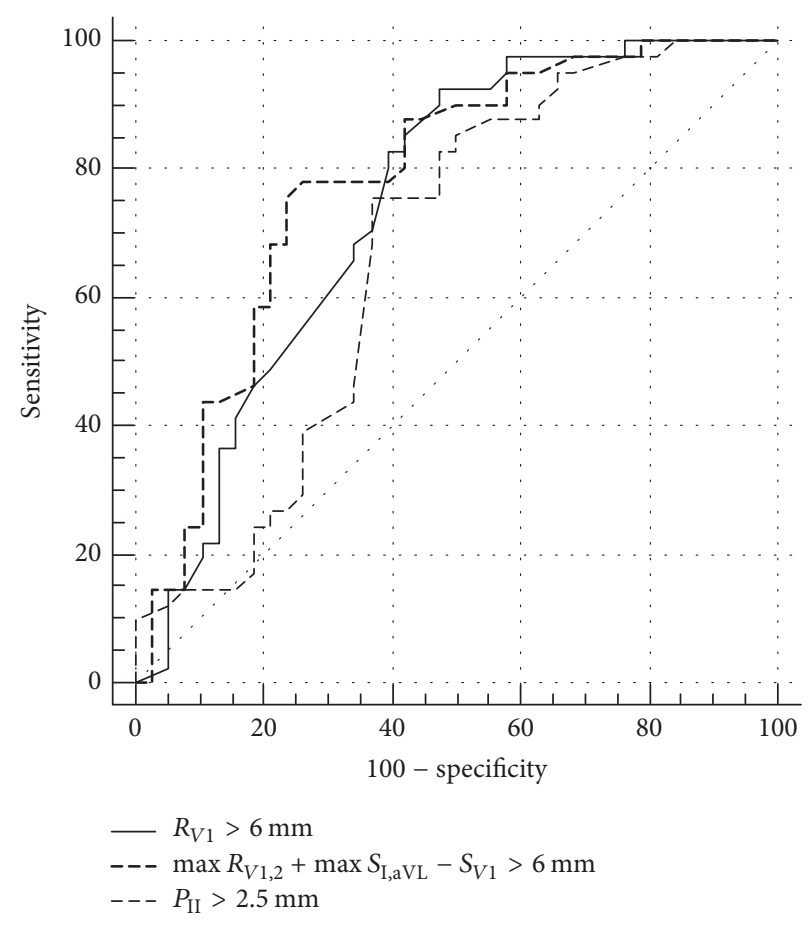

FigURE 2: Electrocardiographic predictors of hemodynamic improvement. $\Delta R_{V 1}$ (AUC: $0.75,95 \%$ CI: $0.63-0.84, p=0.0005$ ), $\Delta$ max $R_{V 1,2}+$ $\max S_{\mathrm{I}, \mathrm{aVL}}-S_{V 1}\left(\right.$ AUC: $0.73,95 \%$ CI: $0.63-0.82, p<0.0001$ ), and $\Delta P_{\mathrm{II}}(\mathrm{AUC:} 0.67,95 \%$ CI: $0.56-0.77, p=0.007$ ).

$(r=0.48, p<0.01)$ correlated with cardiac index. In another study Cheng at al. assessed the relationship between ECG patterns and hemodynamics in 194 IPAH patients [7]. The study showed correlations between $P_{\mathrm{II}}$ and $\mathrm{mPAP}(r=0.35$, $p \leq 0.001)$ and $\mathrm{CI}(r=-0.22, p=0.002) ; R_{V 1}$ and mPAP $(r=0.36, p \leq 0.001) ; S_{V 6}$ and $\mathrm{mPAP}(r=0.26, p=0.03)$ and $\mathrm{CI}(r=-0.22, p=0.003)$. These studies made the background for the hypothesis that ECG can be a valuable tool to monitor hemodynamics in $\mathrm{PH}$. The correlations between changes in $\Delta R_{V 1}, \Delta \max R_{V 1,2}+\max S_{\mathrm{I}, \mathrm{aVL}}-S_{V 1}$, and $\Delta P_{\mathrm{II}}$ and hemodynamic parameters were relatively low. Therefore other factors modifying this relationship should be considered, such as diverse dynamics of reversed RV remodeling in response to improving hemodynamics in individual patients.

4.3. ECG Changes after Mechanical Reduction of Right Ventricle Overload. ECG changes after reduction of right ventricular overload were well shown in CTEPH patients treated with pulmonary endarterectomy (PEA) and in patients operated for right ventricular outflow tract obstruction (RVOTO). In one cohort of 99 patients with CTEPH who underwent pulmonary endarterectomy (PEA), the decrease of $P_{\mathrm{II}}, R_{V 1}$ and normalization of negative $T_{V 1-V 3}$ were observed 1 month after PEA. Additional changes such as increase of $S_{V 1}$, increase of $R: S_{V 6}$, and decreased prevalence of $S_{I} Q_{\text {III }}$ pattern were observed at 1-year follow-up [30]. Of these changes only a decrease in $P_{\mathrm{II}}$ correlated with lowering of MPAP and PVR. In another study of 30 patients who underwent percutaneous pulmonary valve implantation due to severe RVOTO, the improvement in some ECG patterns such as reduction of $R_{\mathrm{aVR}}, R_{V 1}, S_{V 5}, S_{V 6}$, and Sokolow-Lyon index correlated with regression in RV mass and volume [9].

4.4. Changes of ECG after Targeted Treatment for PAH. So far, only two studies have assessed ECG changes after PAH targeted therapy. In first of them Henkens et al. [31] assessed the relationship between hemodynamic response to treatment and changes of selected ECG parameters such as heart rate, $P$ wave amplitude, QRS axis and duration, and $T$ wave axis in $81 \mathrm{PAH}$ patients. Hemodynamic response was defined as a decrease of PVR to less than 500 dyne $\cdot s \cdot \mathrm{cm}^{-5}$. Within 13.1 months of treatment, the responders had lower values of heart rate and $P$ wave amplitude, less rightwards oriented QRS axis, and more rightwards $T$ wave axis than 
the nonresponders. However in another small study of $36 \mathrm{PAH}$ patients, changes in none of the assessed ECG patterns (heart rate, QRS duration, calculated QT interval, premature ventricular contractions, right axis deviation, right bundle branch block, and some measurements of right ventricular hypertrophy) predicted hemodynamic improvement [32]. The aforementioned studies analyzed only a few selected ECG criteria and each of them showed different results. In the present paper we focused on a set of ECG parameters indicating RV overload which were recommended for clinical use in the AHA/ACCF/HRS guidelines [10]. We showed that among them only $R_{V 1}, P_{\mathrm{II}}$, and $\Delta \max R_{V 1,2}+\max S_{\mathrm{I}, \mathrm{aVL}}-S_{V 1}$ are vulnerable to significant changes when hemodynamics of pulmonary circulation improves. We showed that among them only $R_{V 1}, P_{\mathrm{II}}$, and $\Delta \max R_{V 1,2}+\max S_{\mathrm{I}, \mathrm{aVL}}-S_{V 1}$ differentiated patients with and without hemodynamics improvement as presented in Table 3 .

In previous studies on the association between ECG changes and hemodynamics in $\mathrm{PAH}$ both patients with and without RBBB were enrolled. RBBB, however, changes several patterns in ECG and therefore we supposed that it also could modulate the response of ECG to changing hemodynamics. When analyzing patients with $\mathrm{RBBB}$ and without $\mathrm{RBBB}$ separately we found that our data refer only to the group without $\mathrm{RBBB}$ and consequently that changes in the AHA/ACCF/HRS markers of RV overload are not useful to monitor hemodynamics in PAH patients with RBBB.

In our previous study [2] we showed that $R_{V 1}$ and $P_{\text {II }}$ were directly related to right ventricular hypertrophy; therefore we think that changes of these parameters during treatment reflect reverse RV remodeling in patients with significant improvement of hemodynamics. The pharmacological effectiveness on reversal of RV hypertrophy in pulmonary hypertension has been shown in animal models [33-35] and in humans treated with pharmacological [36-39] and surgical treatment [30].

\section{Strengths and Limitations}

The main strength of our study is a comprehensive analysis of changes in several ECG patterns of right ventricular hypertrophy and overload in patients with precapillary pulmonary hypertension treated with targeted therapies. We showed a set of parameters $\left(R_{V 1}, \max R V_{1,2}+\max S_{\mathrm{I}, \mathrm{aVL}}-\right.$ $S_{V 1}$, and $P_{\mathrm{II}}$ ) which can be used in clinical practice to predict hemodynamic effectiveness of treatment. This can be useful in planning the management of $\mathrm{PH}$ patients. We have also shown that the significance of ECG criteria should be interpreted differently in patients with and without RBBB. Additionally we showed that dynamics of ECG in patients with $\mathrm{PH}$ can predict prognosis in this group.

Our study has also some limitations. We included patients with PAH of different etiologies and inoperable CTEPH. However, the pathomechanism of RV overload in both conditions is similar. Nevertheless we cannot refer our conclusions to other types of pulmonary hypertension such as $\mathrm{PH}$ due to left heart disease or due to pulmonary diseases.

\section{Conclusions}

Electrocardiogram may be useful in predicting hemodynamic effects of targeted therapy in precapillary pulmonary hypertension. Decrease of $R_{V 1}, \max R_{V 1,2}+\max S_{\mathrm{I}, \mathrm{aVL}}-S_{V 1}$, and $P_{\mathrm{II}}$ correspond with hemodynamic improvement after treatment. Of these changes a decrease of $R$ wave amplitude in $V_{1}$ is associated with better survival.

\section{Conflicts of Interest}

The authors declare no competing financial interests.

\section{Supplementary Materials}

In supplementary Table S1 we showed how the following ECG parameters changed after $\mathrm{PAH}$ specific treatment in patients with and without significant hemodynamic improvement: $\mathrm{qR}_{V 1}, \mathrm{RSR}_{V 1}, S>R$ in I, $S>R$ in II, $S>R$ in III, $S_{\mathrm{I}}$ and $Q_{I I I}, R: S_{V 1}>R: S_{V 3,4}$, and negative $T$-wave $V_{1}$ through $V_{3}$. In supplementary Tables S2-S5 we compared patients with and without hemodynamic improvement with respect to changes in several ECG parameters after $\mathrm{PAH}$ specific treatment separately for a subgroup with and without RBBB. Table S1: changes of qualitative electrocardiographic parameters after $\mathrm{PAH}$ specific treatment in the whole sample. Table S2: changes of quantitative electrocardiographic parameters after PAH specific treatment in patients without right bundle branch block. Table S3: changes of qualitative electrocardiographic parameters after $\mathrm{PAH}$ specific treatment in patients without right bundle branch block. Table S4: changes of quantitative electrocardiographic parameters after PAH specific treatment in patients with right bundle branch block. Table S5: changes of qualitative electrocardiographic parameters after PAH specific treatment in patients with right bundle branch block. (Supplementary Materials)

\section{References}

[1] N. Galie, M. Humbert, and J. L. Vachiery, "ESC/ERS Guidelines for the diagnosis and treatment of pulmonary hypertension: The Joint Task Force for the Diagnosis and Treatment of Pulmonary Hypertension of the European Society of Cardiology (ESC) and the European Respiratory Society (ERS): Endorsed by: Association for European Paediatric and Congenital Cardiology (AEPC), International Society for Heart and Lung Transplantation (ISHLT)," European Heart Journal, vol. 37, no. 1, pp. 67-119, 2016.

[2] G. Kopeć, A. Tyrka, T. Miszalski-Jamka et al., "Electrocardiogram for the diagnosis of right ventricular hypertrophy and dilation in idiopathic pulmonary arterial hypertension," Circulation Journal, vol. 76, no. 7, pp. 1744-1749, 2012.

[3] G. Kopeć, D. Moertl, T. Miszalski-Jamka et al., "Left ventricular mass is preserved in patients with idiopathic pulmonary arterial hypertension and eisenmenger's syndrome," Heart, Lung and Circulation, vol. 23, no. 5, pp. 454-461, 2014.

[4] G. B. Myers, H. A. Klein, and B. E. Stofer, "The electrocardiographic diagnosis of right ventricular hypertrophy," American Heart Journal, vol. 35, no. 1, pp. 1-40, 1948. 
[5] M. Sokolow and T. P. Lyon, “The ventricular complex in right ventricular hypertrophy as obtained by unipolar precordial and limb leads," American Heart Journal, vol. 38, no. 2, pp. 273-294, 1949.

[6] K. E. Bove, D. T. Rowlands, and R. C. Scott, "Observations on the assessment of cardiac hypertrophy utilizing a chamber partition technique., Circulation, vol. 33, no. 4, pp. 558-568, 1966.

[7] X.-L. Cheng, J.-G. He, Z.-H. Liu et al., "The Value of the Electrocardiogram for Evaluating Prognosis in Patients with Idiopathic Pulmonary Arterial Hypertension," Lung, vol. 195, no. 1, pp. 139-146, 2017.

[8] N. Kanemoto, "Electrocardiographic and Hemodynamic Correlations in Primary Pulmonary Hypertension," Angiology, vol. 39, no. 9, pp. 781-787, 1988.

[9] E. Piotrowicz, E. K. Biernacka, M. Mazgaj et al., "Electrocardiographic characteristics of the right ventricle following hemodynamic improvement after percutaneous pulmonary valve implantation, one year follow-up," Journal of Electrocardiology, vol. 47, no. 5, pp. 612-617, 2014.

[10] E. W. Hancock, B. J. Deal, D. M. Mirvis, P. Okin, P. Kligfield, and L. S. Gettes, "AHA/ACCF/HRS Recommendations for the Standardization and Interpretation of the Electrocardiogram. Part V: Electrocardiogram Changes Associated With Cardiac Chamber Hypertrophy A Scientific Statement From the American Heart Association Electrocardiography and Arrhythmias Committee, Council on Clinical Cardiology; the American College of Cardiology Foundation; and the Heart Rhythm Society," Journal of the American College of Cardiology, vol. 53, no. 11, pp. 992-1002, 2009.

[11] B. Surawicz, R. Childers, B. J. Deal, and L. S. Gettes, "AHA/ACCF/HRS Recommendations for the Standardization and Interpretation of the Electrocardiogram. Part III: Intraventricular Conduction Disturbances A Scientific Statement From the American Heart Association Electrocardiography and Arrhythmias Committee, Council on Clinical Cardiology; the American College of Cardiology Foundation; and the Heart Rhythm Society," Journal of the American College of Cardiology, vol. 53, no. 11, pp. 976-981, 2009.

[12] R. D. Mosteller, "Simplified calculation of body-surface area," The New England Journal of Medicine, vol. 317, no. 17, article 1098, 1987.

[13] K. Al-Naamani, T. Hijal, V. Nguyen, S. Andrew, T. Nguyen, and T. Huynh, "Predictive values of the electrocardiogram in diagnosing pulmonary hypertension," International Journal of Cardiology, vol. 127, no. 2, pp. 214-218, 2008.

[14] P. M. Butler, S. I. Leggett, C. M. Howe, C. J. Freye, N. B. Hindman, and G. S. Wagner, "Identification of electrocardiographic criteria for diagnosis of right ventricular hypertrophy due to mitral stenosis," American Journal of Cardiology, vol. 57, no. 8, pp. 639-643, 1986.

[15] T. Hiroki, K. Arakawa, J. Muramatsu et al., "New electrocardiographic criteria for diagnosing right ventricular hypertrophy in mitral stenosis - Comparison with Bonner's and Mortara's Criteria," Japanese Circulation Journal, vol. 52, no. 10, pp. 11141120, 1988.

[16] J. V. Behar, C. M. Howe, N. B. Wagner et al., "Performance of new criteria for right ventricular hypertrophy and myocardial infarction in patients with pulmonary hypertension due to cor pulmonale and mitral stenosis," Journal of Electrocardiology, vol. 24, no. 3, pp. 231-237, 1991.
[17] J. Lehtonen, S. Sutinen, M. Ikaheimo, and P. Paakko, "Electrocardiographic criteria for the diagnosis of right ventricular hypertrophy verified at autopsy," CHEST, vol. 93, no. 4, pp. 839842, 1988.

[18] M. L. Murphy, P. N. Thenabadu, N. de Soyza et al., "Reevaluation of electrocardiographic criteria for left, right and combined cardiac ventricular hypertrophy," American Journal of Cardiology, vol. 53, no. 8, pp. 1140-1147, 1984.

[19] D. Bonderman, P. Wexberg, A. M. Martischnig et al., "A noninvasive algorithm to exclude pre-capillary pulmonary hypertension," European Respiratory Journal, vol. 37, no. 5, pp. 1096-1103, 2011.

[20] N. Kanemoto, "Electrocardiogram in primary pulmonary hypertension," European Heart Journal, vol. 12, no. 3-4, pp. 181193, 1980.

[21] E. Bossone, G. Paciocco, D. Iarussi et al., "The prognostic role of the ECG in primary pulmonary hypertension," CHEST, vol. 121, no. 2, pp. 513-518, 2002.

[22] N. Kanemoto, "Electrocardiogram in primary pulmonary hypertension - With special reference to prognosis," The Tokai Journal of Experimental and Clinical Medicine, vol. 12, no. 3, pp. 173-179, 1987.

[23] M. Waligóra, G. Kopeć, K. Jonas et al., "Mechanism and prognostic role of $\mathrm{qR}$ in $\mathrm{V} 1$ in patients with pulmonary arterial hypertension," Journal of Electrocardiology, vol. 50, no. 4, pp. 476-483, 2017.

[24] F. F. Hildenbrand, I. Fauchère, L. C. Huber, S. Keusch, R. Speich, and S. Ulrich, "A low resting heart rate at diagnosis predicts favourable long-term outcome in pulmonary arterial and chronic thromboembolic pulmonary hypertension. A prospective observational study," Respiratory Research, vol. 13, article no. 76, 2012.

[25] D. Bandorski, H. Bogossian, A. Ecke et al., "Evaluation of the prognostic value of electrocardiography parameters and heart rhythm in patients with pulmonary hypertension," Cardiology Journal, vol. 23, no. 4, pp. 465-472, 2016.

[26] W. J. Cantor, D. A. Harrison, J. S. Moussadji et al., "Determinants of survival and length of survival in adults with Eisenmenger syndrome," American Journal of Cardiology, vol. 84, no. 6, pp. 677-681, 1999.

[27] P.-Y. Sun, X. Jiang, M. Gomberg-Maitland et al., "Prolonged QRS duration: a new predictor of adverse outcome in idiopathic pulmonary arterial hypertension," CHEST, vol. 141, no. 2, pp. 374-380, 2012.

[28] J. D. Rich, T. Thenappan, B. Freed et al., "QTc prolongation is associated with impaired right ventricular function and predicts mortality in pulmonary hypertension," International Journal of Cardiology, vol. 167, no. 3, pp. 669-676, 2013.

[29] A. R. Tonelli, M. Baumgartner, L. Alkukhun, O. A. Minai, and R. A. Dweik, "Electrocardiography at diagnosis and close to the time of death in pulmonary arterial hypertension," Annals of Noninvasive Electrocardiology, vol. 19, no. 3, pp. 258-265, 2014.

[30] S. Ghio, A. Turco, C. Klersy et al., "Changes in surface electrocardiogram in patients with chronic thromboembolic pulmonary hypertension undergoing pulmonary endarterectomy. Correlations with hemodynamic and echocardiographic improvements after surgery," Journal of Electrocardiology, vol. 49, no. 2, pp. 223-230, 2016.

[31] I. R. Henkens, C. T.-J. Gan, S. A. Van Wolferen et al., "ECG monitoring of treatment response in pulmonary arterial hypertension patients," CHEST, vol. 134, no. 6, pp. 1250-1257, 2008. 
[32] D. Snipelisky, C. Burger, B. Shapiro, and F. Kusumoto, "Electrocardiographic changes in patients responding to treatment with group i pulmonary arterial hypertension," Southern Medical Journal, vol. 106, no. 11, pp. 618-623, 2013.

[33] M. A. Cavasin, K. M. Demos-Davies, K. B. Schuetze et al., "Reversal of severe angioproliferative pulmonary arterial hypertension and right ventricular hypertrophy by combined phosphodiesterase- 5 and endothelin receptor inhibition," Journal of Translational Medicine, vol. 12, no. 1, article no. 314, 2014.

[34] O. Rafikova, R. Rafikov, S. Kumar et al., "Bosentan inhibits oxidative and nitrosative stress and rescues occlusive pulmonaryhypertension," Free Radical Biology \& Medicine, vol. 56, pp. 28-43, 2013.

[35] M. Iglarz, K. Landskroner, Y. Bauer et al., "Comparison of macitentan and bosentan on right ventricular remodeling in a rat model of non-vasoreactive pulmonary hypertension," Journal of Cardiovascular Pharmacology, vol. 66, no. 5, pp. 457467, 2015.

[36] S. Rich and B. H. Brundage, "High-dose calcium channelblocking therapy for primary pulmonary hypertension: Evidence for long-term reduction in pulmonary arterial pressure and regression of right ventricular hypertrophy," Circulation, vol. 76, no. 1, pp. 135-141, 1987.

[37] A. L. Hinderliter, P. W. Willis IV, R. J. Barst et al., "Effects of long-term infusion of prostacyclin (epoprostenol) on echocardiographic measures of right ventricular structure and function in primary pulmonary hypertension," Circulation, vol. 95, no. 6, pp. 1479-1486, 1997.

[38] A. R. Tonelli, D. Conci, B. K. Tamarappoo, J. Newman, and R. A. Dweik, "Prognostic value of echocardiographic changes in patients with pulmonary arterial hypertension receiving parenteral prostacyclin therapy," Journal of the American Society of Echocardiography, vol. 27, no. 7, pp. 733-e2, 2014.

[39] N. Galiè, A. L. Hinderliter, A. Torbicki et al., "Effects of the oral endothelin-receptor antagonist bosentan on echocardiographic and doppler measures in patients with pulmonary arterial hypertension," Journal of the American College of Cardiology, vol. 41, no. 8, pp. 1380-1386, 2003. 


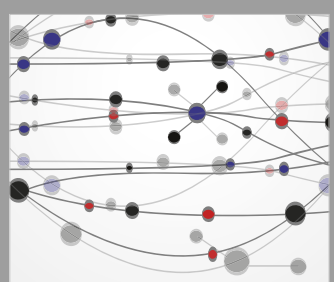

The Scientific World Journal
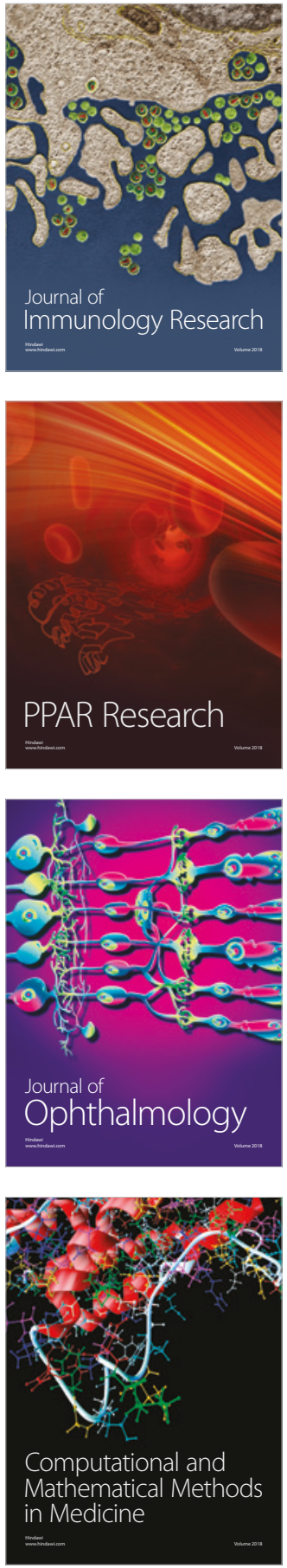

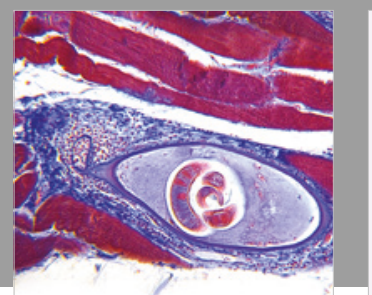

Gastroenterology Research and Practice

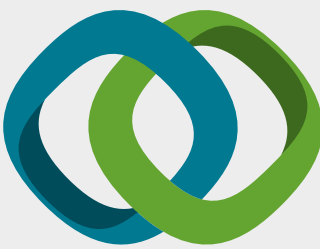

\section{Hindawi}

Submit your manuscripts at

www.hindawi.com
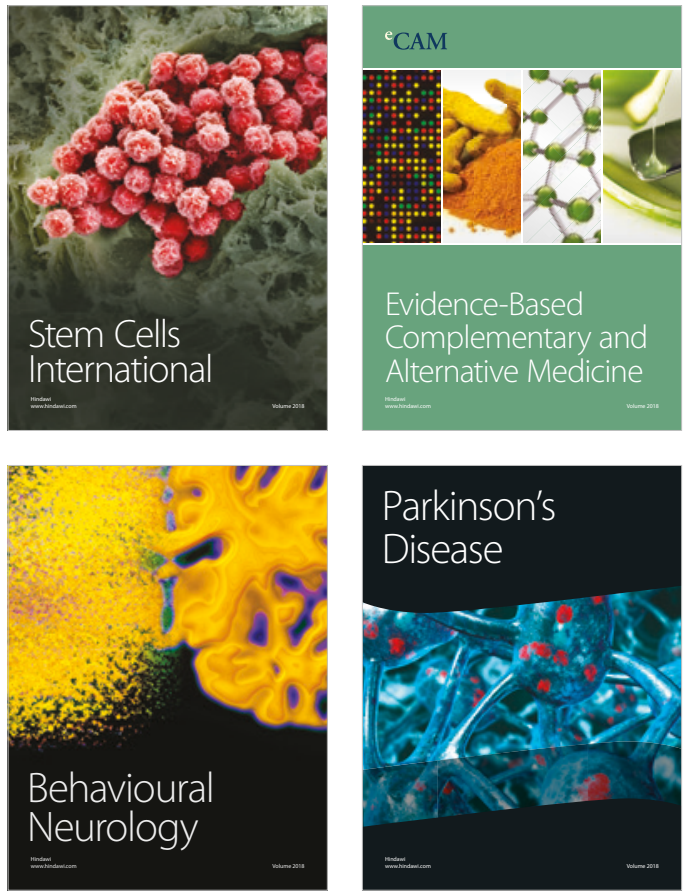

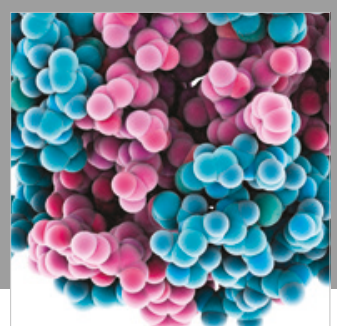

ournal of

Diabetes Research

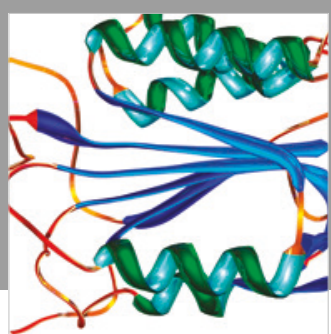

Disease Markers
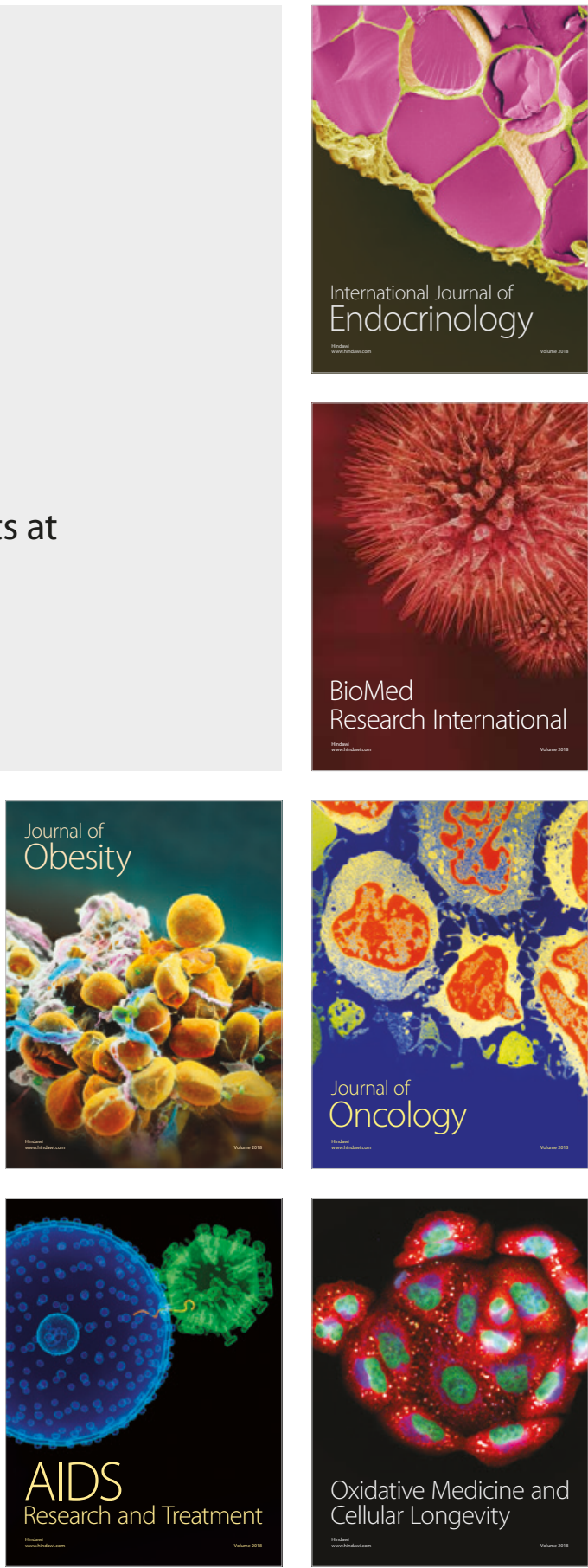